\title{
Can osseous landmarks in the distal medial humerus be used to identify the attachment sites of ligaments and tendons: paleopathologic-anatomic imaging study in cadavers
}

\author{
Florian M. Buck • Cristiane S. Zoner • Fabiano Cardoso • Ramon Gheno • \\ Marcelo A. C. Nico • Debra J. Trudell • Tori D. Randall • Donald Resnick
}

Received: 29 July 2009 /Revised: 24 August 2009 /Accepted: 26 August 2009 / Published online: 10 October 2009

(C) The Author(s) 2009. This article is published with open access at Springerlink.com

\begin{abstract}
Objective To describe osseous landmarks that allow identification of the attachments of the ligaments and tendons in the distal medial aspect of the humerus.

Materials and methods Reliable osseous landmarks in the distal medial aspect of the humerus were identified in 34 well-preserved specimens from a paleopathologic collection. These osseous landmarks were then sought in magnetic resonance (MR) images of ten cadaveric elbow specimens so that the ease of their visualization and optimal imaging plane could be assessed. To assign these osseous landmarks to specific attachments of the tendons and ligaments in the distal medial humerus, we cut the specimens in slices and photographed and examined them. Subsequently, the prevalence of these osseous landmarks as well as the attachment sites of the tendons and ligaments in this location was determined.

Results We determined ten reliable osseous landmarks in the distal medial aspect of the humerus, their prevalence
\end{abstract}

F. M. Buck $\cdot$ C. S. Zoner $\cdot$ F. Cardoso $\cdot$ R. Gheno

M. A. C. Nico $\cdot$ D. J. Trudell $•$ D. Resnick

Department of Radiology,

Veterans Administration Medical Center,

San Diego, CA, USA

F. M. Buck $(\bowtie)$

Uniklinik Balgrist, Institut für Diagnostische Radiologie,

Forchstrasse 340,

8008 Zurich, Switzerland

e-mail: florian.buck@balgrist.ch

T. D. Randall

Physical Anthropology, San Diego Museum of Man,

San Diego, CA, USA and ease of identification, and their relationship to the attachments of the tendons and ligaments at the medial distal humerus.

Conclusion It is possible to use osseous landmarks at the distal medial humerus to facilitate identification of the different attachments of tendons and ligaments when MR images of the elbow are assessed.

Keywords Elbow $\cdot$ Magnetic resonance imaging $\cdot$ Medial epicondyle $\cdot$ Attachment $\cdot$ Tendon $\cdot$ Ligament $\cdot$ Anatomy

\section{Introduction}

The anatomy of the distal medial aspect of the humerus, especially at and around the medial epicondyle, is complex, because of the close relationships of many tendons and ligaments that attach to this area. The assessment of pathologic abnormalities in this region is even more difficult, because the assignment of alterations in magnetic resonance (MR) images to a specific tendon or ligament is challenging. Because pathologic processes further impede soft tissue visualization, it would be very helpful to define a set of osseous landmarks that would allow identification of individual structures. Although many publications have described the general anatomy of the medial aspect of the distal humerus [1-10], as well as normal MR imaging findings and those in multiple pathologic conditions $[9,11-18]$, there is no publication that defines in great detail those bone landmarks that are indicative of the attachments of the tendons and ligaments that are found in this region. Such an approach, which has been used successfully to investigate the attachments of the rotator cuff tendons in the greater tuberosity of the humerus, was the purpose of this investigation. 


\section{Materials and methods}

This cadaveric study did not require institutional review board approval according to the rules of the hospital where the study was performed.

\section{Paleopathologic investigation}

Two fellowship-trained musculoskeletal radiologists thoroughly evaluated in consensus the distal medial surface of the humerus in 34 well-preserved humerus specimens from the collection of a local museum. Constant osseous landmarks, seen as prominences, crests, sulci, and flattened areas of the bone surface, were identified. Their prevalence was recorded, and their detectability on physical inspection was rated from 1 to 4 on a continuous scale: 1, not detectable; 2, poor detectability; 3, fair detectability; and 4, good detectability. If there were pathologic proliferative bone changes in any of these areas, their locations were also recorded.

\section{Cadavers and specimen preparation}

Ten fresh human elbow specimens were harvested from nine nonembalmed cadavers (two women, three men, four unknown; age range at death 62-98 years; mean age at death 80.2 years) and used according to institutional guidelines, with written informed consent having been given before death. The specimens included the distal half of the arm, the elbow joint, and the proximal half of the forearm. All specimen were immediately deep-frozen at $-40^{\circ} \mathrm{C}$ (Forma Bio-Freezer; Forma Scientific, Marietta, $\mathrm{OH}, \mathrm{USA}$ ). Before undergoing MR imaging, all specimens were allowed to thaw for $24 \mathrm{~h}$ at room temperature.

\section{MR imaging}

MR Imaging was done with a 1.5 T MR system (Signa; GE Healthcare Technologies, Milwaukee, WI, USA). An eightchannel, receive-only, general-purpose flex coil (Flex coil small; Medical Advances, Milwaukee, WI, USA) was employed. The coil was wrapped around the elbow specimen, with the center of the coil at the level of the medial humeral epicondyle. The specimen was then placed fully extended and in maximal supination in the gantry, analogous to the supine position of a patient. Proton density-weighted non-fat saturated spin-echo sequences [repetition time 3,000 ms; echo time $13 \mathrm{~ms}$; field of view (FOV) $10 \mathrm{~cm} \times 10 \mathrm{~cm}$; matrix 512 pixels $\times 512$ pixels; number of excitations 4; slice thickness $2 \mathrm{~mm}$; interslice spacing $0 \mathrm{~mm}$; echo train length = turbo factor, 7 ; bandwidth, $\pm 31 \mathrm{kHz}$ ] were acquired in the transverse, sagittal, and coronal planes. Additionally, a proton density-weighted fat-saturated spin-echo sequence (repetition time 3,000 ms; echo time $13 \mathrm{~ms}$; FOV $10 \mathrm{~cm} \times 10 \mathrm{~cm}$; matrix 512 pixels $\times$ 512 pixels; number of excitations 4; slice thickness $2 \mathrm{~mm}$; interslice spacing $0 \mathrm{~mm}$; echo train length = turbo factor, 7; bandwidth, $\pm 31 \mathrm{kHz}$ ) was performed in the coronal plane.

All the osseous landmarks defined by the paleopathologic investigation were sought on the MR images from the cadavers by two fellowship-trained radiologists in consensus. The visibility of the osseous landmarks was rated on the same scale (1, not detectable; 2 , poor detectability; 3 , fair detectability; and 4, good detectability) as was utilized for the paleopathologic evaluation. The best imaging plane was assessed for each structure. If proliferative bone changes were evident, their location was recorded.

\section{Radiologic-anatomic correlation}

Directly after imaging, the specimens were frozen again at $-40^{\circ} \mathrm{C}$ for at least 3 days. All specimens were then cut with a band saw into $4 \mathrm{~mm}$-thick slices. Four specimens were cut in the coronal plane, three in the axial plane, and three in the sagittal plane, all planes corresponding exactly to those of the MR images. Photographs and plain films (Faxitron HP 43805N X-Ray System, Hewlett-Packard, Palo Alto, CA, USA; tube current $30 \mathrm{kV}$; exposure time $30 \mathrm{~s})$ of all sections were obtained.

Two fellowship-trained musculoskeletal radiologists identified in consensus all ligaments and tendons arising
Fig. 1 Illustration of the different osseous landmarks and areas examined in the distal medial aspect of the humerus. Note: in this example there is a type 2 anterosuperior tubercle and a T-shaped intertubercular sulcus

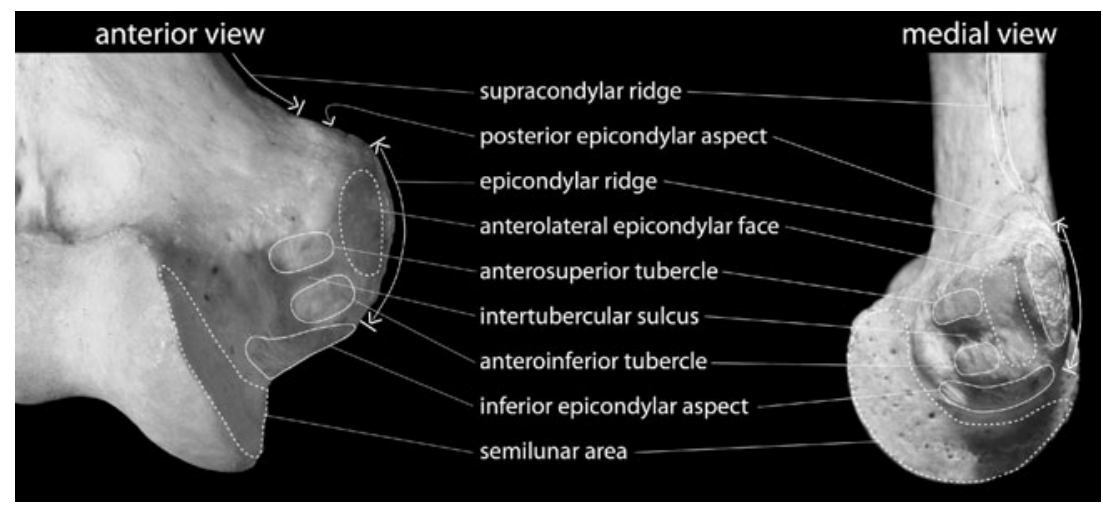




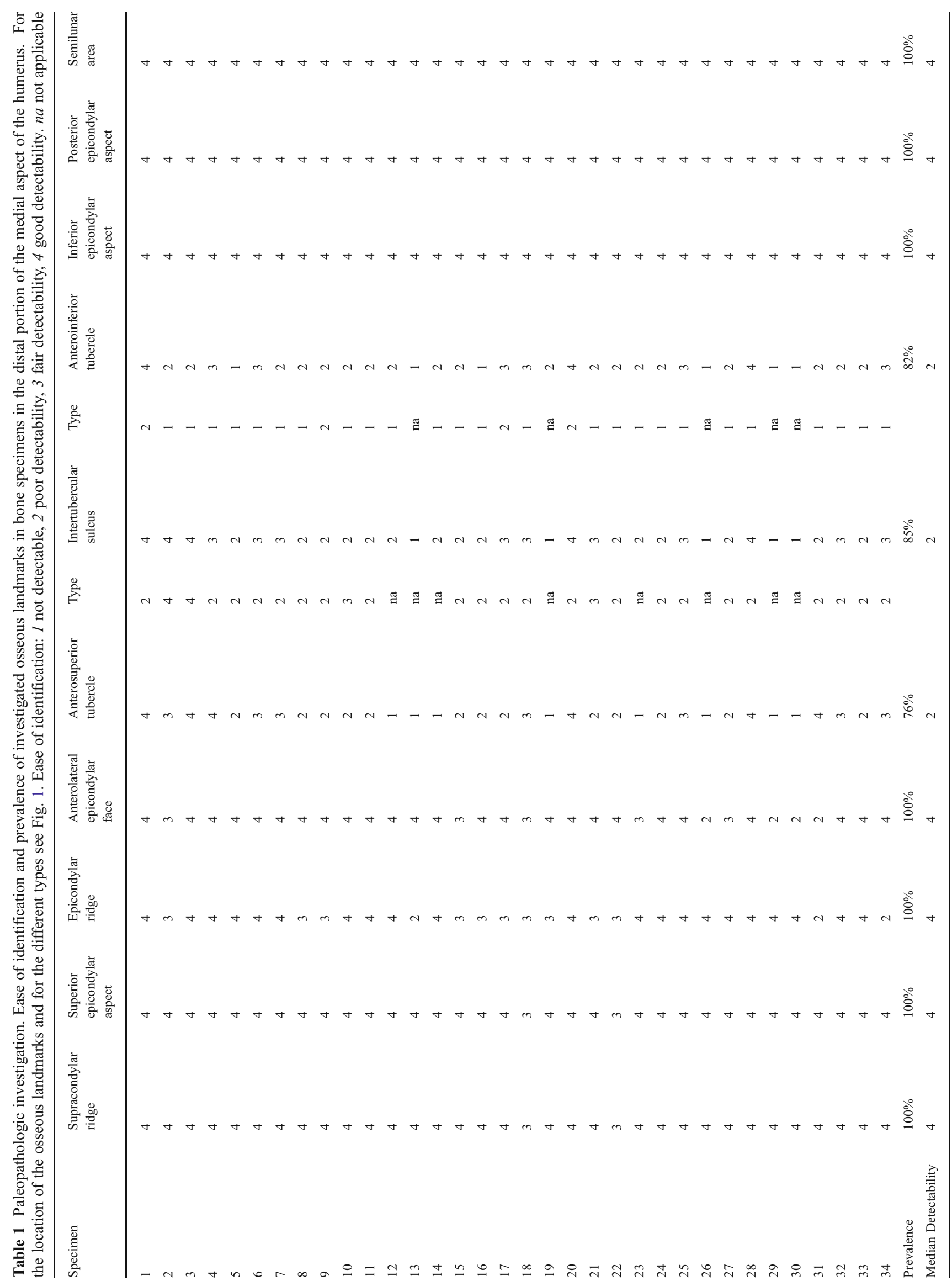


from the distal medial surface of the humerus. The precise location of their attachments was investigated, with attention being paid to the anatomic features at the regions of tendon and ligament attachment. The best imaging plane was identified. All proliferative bone changes found in the paleopathologic investigation, MR images, and anatomical inspection were classified as osteophytes or enthesophytes, based on their localization. Osteophytes were defined as proliferative bone changes adjacent to the articular cartilage. Enthesophytes were defined as proliferative bone changes at the regions of tendon and ligament attachment.

In a final step the locations of the osseous landmarks that resulted from paleopathologic evaluation were matched with the observed attachments of the different ligaments and tendons, as observed during the anatomical investigation. Additionally, the plain films of the anatomic specimen sections were used to correlate further the exact locations of osseous landmarks and attachments.

\section{Results}

\section{Paleopathologic investigation}

Ten specific areas were defined in consensus in the medial aspect of the distal portion of the humerus (Fig. 1): supracondylar ridge, superior aspect of the medial epicondyle, epicondylar ridge, anterolateral epicondylar face, anterosuperior and anteroinferior tubercles with the intervening intertubercular sulcus, inferior aspect of the medial epicondyle, posterior aspect of the medial epicondyle, and semilunar area.

Table 2 Location and number of osteophytes and enthesophytes

\begin{tabular}{lll}
\hline $\begin{array}{l}\text { Osseous proliferative } \\
\text { changes }\end{array}$ & $\begin{array}{l}\text { Bone } \\
\text { specimens }\end{array}$ & $\begin{array}{l}\text { Cadaveric } \\
\text { specimens }\end{array}$ \\
\hline $\begin{array}{l}\text { Number of specimens } \\
\text { Enthesophytes }\end{array}$ & 36 & 10 \\
supracondylar ridge & 6 & 0 \\
superior epicondylar aspect & 0 & 0 \\
posterior epicondylar aspect & 0 & 0 \\
epicondylar ridge & 13 & 6 \\
anterolateral epicondylar face & 5 & 0 \\
anterosuperior tubercle & 10 & 0 \\
intertubercular tubercle & 6 & 0 \\
anteroinferior tubercle & 8 & 0 \\
inferior epicondylar aspect & 6 & 0 \\
Osteophytes & & 6 \\
semilunar area & 26 & \\
\hline
\end{tabular}

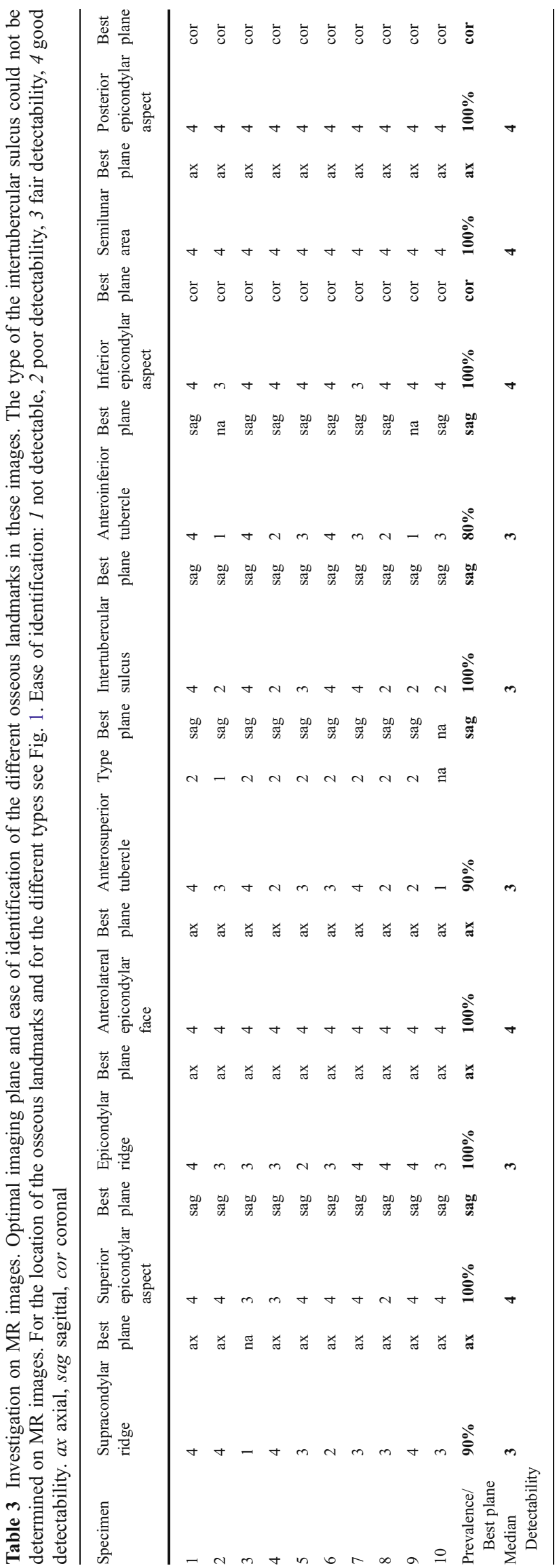


The anterosuperior tubercle could be further classified into one of four types, based on its profile: 1 , round; 2 , elongated in the direction of the adjacent intertubercular sulcus; 3, elongated as in type 2 but with a clear narrowing in its middle; and 4, separation of the tubercle into two smaller tubercles with a minute sulcus between the two. The intertubercular sulcus could be further classified into one of two types, based on its shape: 1, I-shaped; 2, T-shaped, with the T-bar at the base of the epicondyle.

The data related to the detectability of each of the ten specific areas are shown in Table 1. The prevalence of the different osseous landmarks ranged between $76 \%$ and $100 \%$, and the average detectability ranged between 2.2 and 4 . The prevalence of proliferative bone changes are shown in Table 2.

\section{Cadaveric investigation}

The MR imaging results are shown in Table 3. The prevalence of the various osseous landmarks differed slightly from that provided by the paleopathologic investigation (range 80-100\%). Detectability ranged between 2.7 and 4, and, in general, such detectability was lower on the MR images than in the paleopathologic evaluation. The detectability of the anterolateral epicondylar face in the axial plane was greater on the MR images. Furthermore, detectability of the anterosuperior tubercle, anteroinferior tubercle, and intertubercular sulcus was greater on the sagittal MR images than in the paleopathologic evaluation (Tables 1 and 3). It was impossible for us to grade the type of the intertubercular sulcus in all specimens. The locations of the proliferative bone changes are shown in Table 2.

\section{Radiologic-anatomic correlation}

The locations of the different attachments of the tendons and ligaments in the medial aspect of the distal portion of the humerus are presented in Fig. 2. The tendons that constituted the common flexor tendon (flexor carpi radialis, flexor carpi ulnaris, flexor digitorum superficialis, and palmaris longus tendons) were not always separable in the anatomic specimens. In three specimens, however, there were small layers of fat between the tendons of the pronator teres, flexor carpi radialis, and flexor carpi ulnaris tendons, allowing clear identification of these constituents of the common flexor tendon (Fig. 3). Nevertheless, it was possible for us to describe the main attachment sites of the different tendons by evaluating the direction of the most proximal identifiable tendon fibers and using this direction to estimate the tendon diameter at the bone surface.

The medial intermuscular septum inserted in the supracondylar ridge, clearly separated from the origin of the pronator teres tendon. In all specimens there was a gap, void of structures, located between them. The attachment of the pronator teres tendon was located between those of the medial intermuscular septum and the common flexor tendon at the superior aspect of the epicondyle, extending laterally to the epicondylar ridge and extending inferiorly to the summit of the anterosuperior tubercle but never reaching the intertubercular sulcus. The attachment of the common flexor tendon consisted of a broad area at the anterior aspect of the epicondyle between the origin of the pronator teres tendon and the ulnar collateral ligament. It included the epicondylar ridge and anterolateral epicondylar face and extended slightly to the posterior aspect of the epicondyle, becoming intimate with the ulnar nerve and the posterior ulnar recurrent vessels. In sagittal images

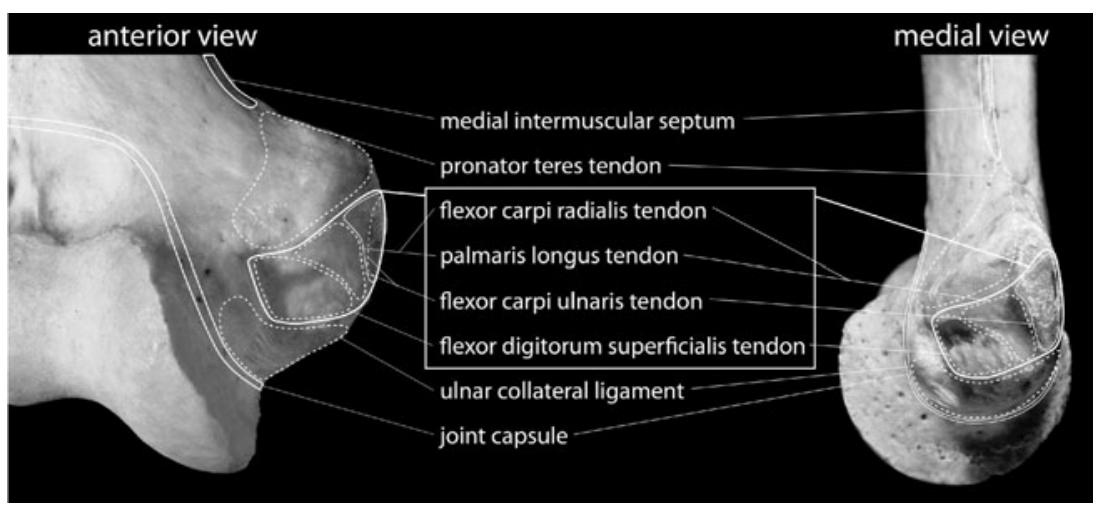

Fig. 2 Attachments in the distal medial aspect of the humerus. Attachments listed in the white box relate to the common flexor tendon. These attachments were not individually distinguishable, and the indicated areas, therefore, are considered to be only the main attachment site of the tendinous fibers. The attachment of the joint capsule was evaluated only anterior, inferior, and posterior to the medial epicondyle, but it is also indicated more proximally in the current literature. Note: for the pronator teres and flexor digitorum superficialis tendons, only those attachments in the humerus have been considered 


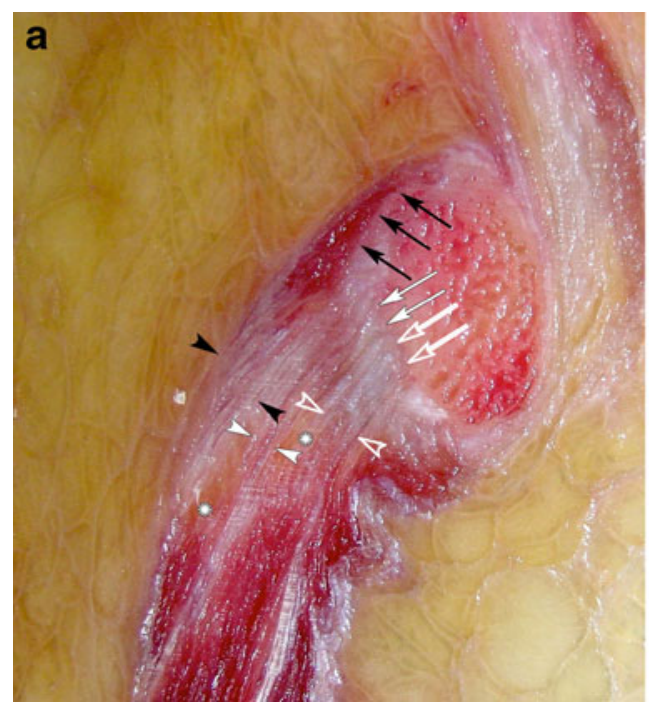

Fig. 3 Attachment site of the pronator teres tendon (black arrowheads) in the anterosuperior aspect of the medial epicondyle from the 12 o'clock to 3 o'clock positions (black arrows). The flexor carpi ulnaris tendon (solid white arrowheads) and flexor carpi radialis tendon (open arrowheads) attach from the 3 o'clock to 5 o'clock

of the epicondyle, with reference to 12 o'clock superiorly and 3 o'clock anteriorly, this attachment included the circumference of the epicondyle from 3 o'clock to 6 o'clock, including the summit of the anterosuperior tubercle, the intertubercular sulcus, and the anteroinferior tubercle. In these sagittal images the attachment of the pronator teres tendon included the circumference of the epicondyle and extended from the 12 o'clock to 3 o'clock positions. The main attachment site of the flexor carpi radialis and palmaris longus tendons included the anterosuperior tubercle and anterolateral epicondylar face. The

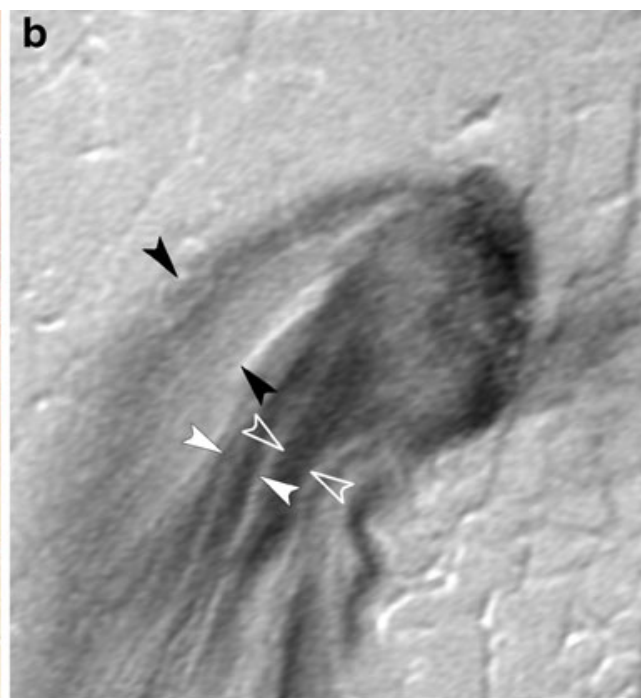

positions (solid white arrows/open white arrows). The tendons are separated by small amounts of fatty tissue (asterisks). a Anatomical slice in the sagittal plane, b corresponding proton density-weighted MR image. Note: the flexor digitorum superficialis tendon attaches more laterally in the base of the epicondyle

main attachment site of the flexor carpi ulnaris tendon was the distal two-thirds of the epicondylar ridge, and the main attachment site of the flexor digitorum superficialis tendon included the intertubercular sulcus and the area of the anteroinferior tubercle (Fig. 4).

The attachment of the ulnar collateral ligament was a broad triangular area in the inferior aspect of the epicondyle, with considerable anterior and posterior extensions, consistent with the extensive data provided by Fuss [2]. The anterior part of the ulnar collateral ligament was identified as a thick hypointense ligament originating in the anterior
Fig. 4 Attachment site of the flexor digitorum superficialis tendon (black arrowheads). Most fibers attach in the intertubercular sulcus (black arrow). Anterior to the flexor digitorum superficialis tendon, the pronator teres tendon is visible (asterisk). a Anatomical in the sagittal plane, b corresponding proton density-weighted MR image
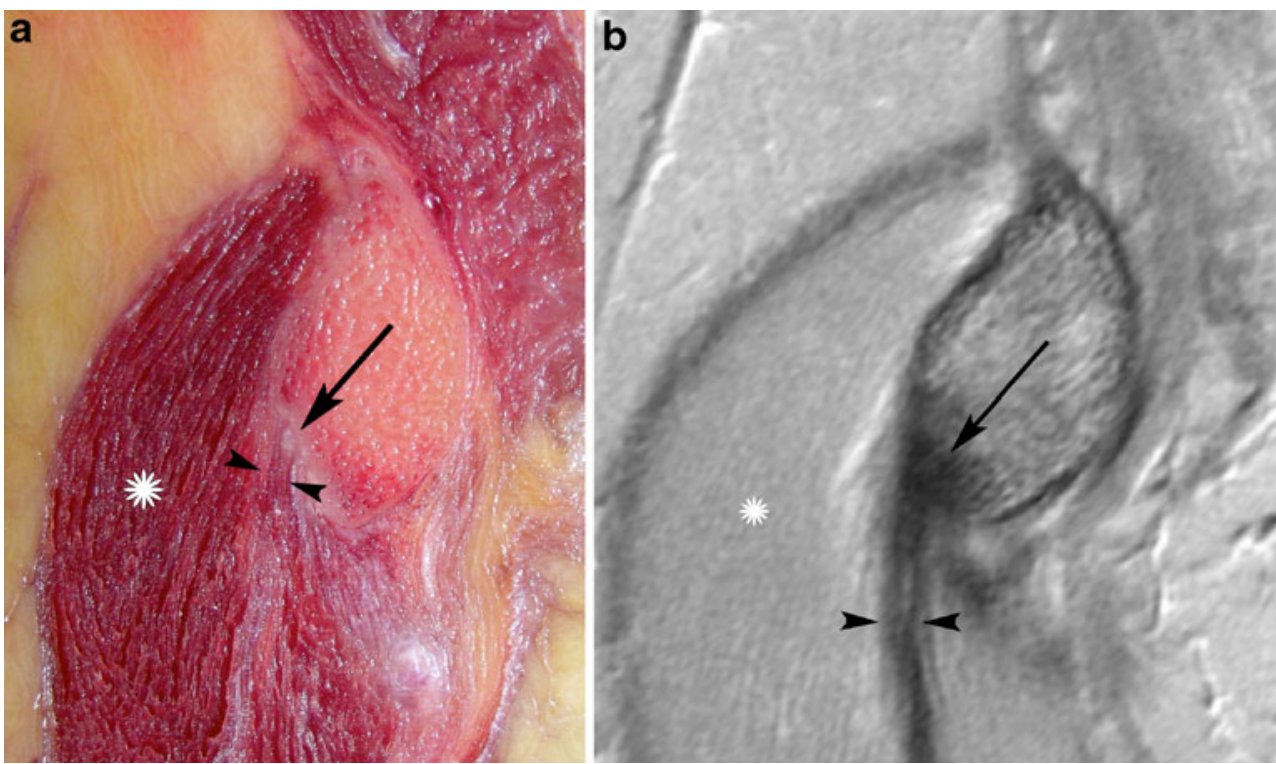


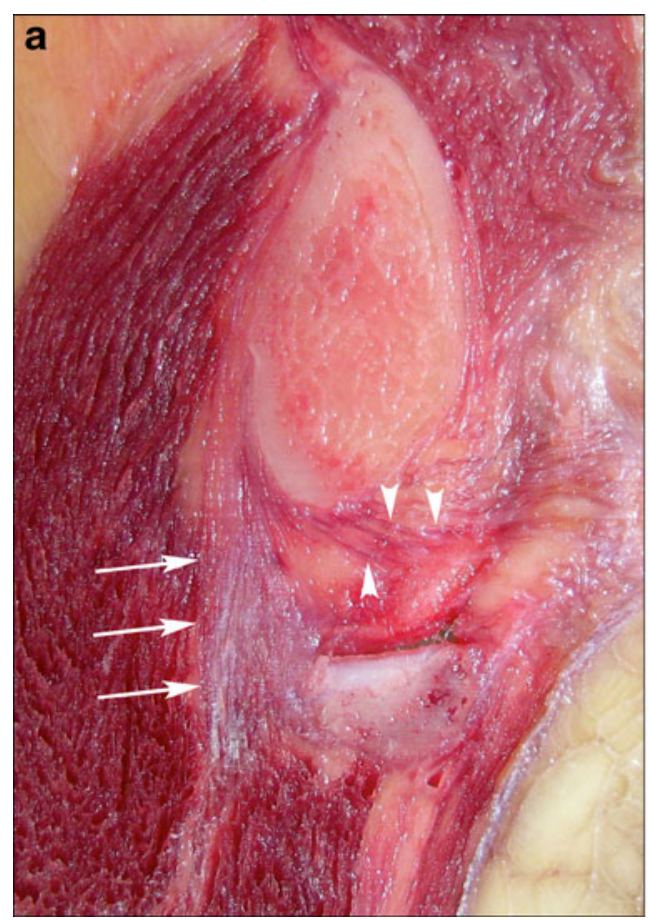

Fig. 5 Attachment of the ulnar collateral ligament. The anterior part is seen as a thick ligament (white arrows), whereas the posterior part consists of multiple fibers with intervening fatty tissue (whites arrowheads). More anteriorly, the flexor digitorum superficialis

half of the attachment (Fig. 5). The posterior part was characterized by multiple ligamentous fibers with interspersed fatty tissue originating in the posterior half of the attachment. The joint capsule was visible only along its course from the anterior aspect to the inferior aspect and,

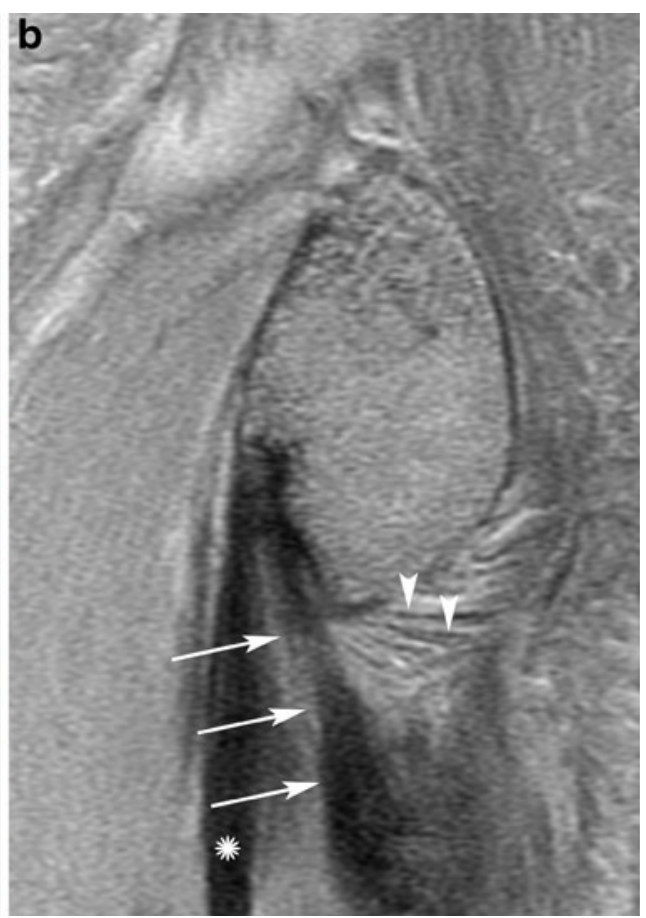

tendon courses upwards to the intertubercular sulcus (asterisk). a Anatomical slice in the sagittal plane, $\mathbf{b}$ corresponding proton densityweighted MR image

finally, to the posterior aspect of the epicondyle. Its attachment was intimate with that of the ulnar collateral ligament. At the inferior aspect of the epicondyle, there was a consistent small fat pad between this ligament and the joint capsule. There were no attachments in the posterior

Table 4 Imaging. Anatomic correlation

\begin{tabular}{|c|c|c|c|}
\hline Attachment/footprint & Osseous landmarks & $\begin{array}{l}\text { Prevalence of the } \\
\text { landmarks }\end{array}$ & $\begin{array}{l}\text { Best imaging } \\
\text { plane }\end{array}$ \\
\hline Medial intermuscular septum & supracondylar ridge & $90 \%$ & axial \\
\hline \multirow[t]{2}{*}{ Pronator teres tendon } & superior epicondylar aspect & $100 \%$ & sagittal \\
\hline & superior margin of anterosuperior tubercle & $90 \%$ & sagittal \\
\hline \multicolumn{4}{|l|}{ Common flexor tendon } \\
\hline \multirow[t]{2}{*}{ Flexor carpi ulnaris tendon } & epicondylar ridge & $100 \%$ & axial \\
\hline & superior margin of anterolateral epicondylar face & $100 \%$ & axial \\
\hline \multirow{2}{*}{$\begin{array}{l}\text { Flexor carpi radialis \& Palmaris } \\
\text { longus tendon }\end{array}$} & anterolateral epicondylar face & $100 \%$ & axial \\
\hline & medial aspect of anterosuperior tubercle & $90 \%$ & sagittal \\
\hline \multirow[t]{3}{*}{ Flexor digitorum superficialis tendon } & lateral aspect of anterosuperior tubercle & $90 \%$ & sagittal \\
\hline & intertubercular sulcus (main insertion site) & $100 \%$ & sagittal \\
\hline & anteroinferior tubercle & $80 \%$ & sagittal \\
\hline Ulnar collateral ligament & inferior epicondylar aspect & $100 \%$ & coronal \\
\hline Joint capsule & $\begin{array}{l}\text { inferior epicondylar aspect lateral to ulnar } \\
\text { collateral ligament }\end{array}$ & $100 \%$ & coronal \\
\hline
\end{tabular}




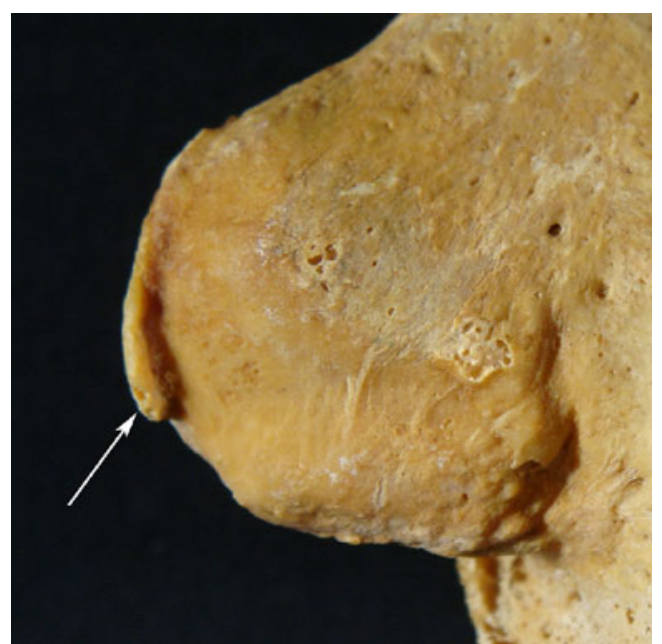

Fig. 6 Paleopathologic specimen from person of unknown age at death. An enthesophyte (arrow) is seen at the attachment site of the flexor carpi ulnaris tendon

aspect of the epicondyle and the semilunar area. None of the reported anatomic variations was evident in our study. The results from the radiologic-anatomic correlation are summarized in Table 4.

Based on the locations of the attachments of the medial tendons and ligaments, proliferative bone changes could be assigned to specific structures and could be classified as shown in Table 2. Enthesophytes were most commonly found in the epicondylar ridge (Figs. 6 and 7), and osteophytes were detected only in the semilunar area, a broad region without any attachment.

\section{Discussion}

When MR images of the elbow are being studied, the precise identification of the individual tendinous and ligamentous origins can be troublesome, making difficult the assignment of a visualized abnormality to a specific tendon or ligament, or, for that matter, its assignment as a tendinous or ligamentous alteration. To find reliable osseous landmarks in the distal medial aspect of the humerus that would allow such assignment, we started our evaluation using well-preserved paleopathologic specimens. These specimens made it possible for us to investigate the osseous structures in great detail. The knowledge derived from these investigations was then applied to MR images of the elbow in fresh cadaveric specimens. This approach allowed us to find even small osseous landmarks that are not constantly seen in clinical MR images.

With this protocol, osseous landmarks could be established that allowed identification of the attachments of the tendons, ligaments, and joint capsule in the distal medial aspect of the humerus, and optimal imaging planes for and grades of identifiability of these landmarks could be determined. As one example, most of the fibers of the common flexor tendon were found to attach in the intertubercular sulcus. The results of our investigation of the attachments of the different osseous landmarks originating in the distal medial aspect of the humerus were in compliance with recent anatomical and radiological publications [1-3, 9-11, 14, 15, 19].

In our investigation enthesophytes were found in many different locations. Based on the large number of entheso-
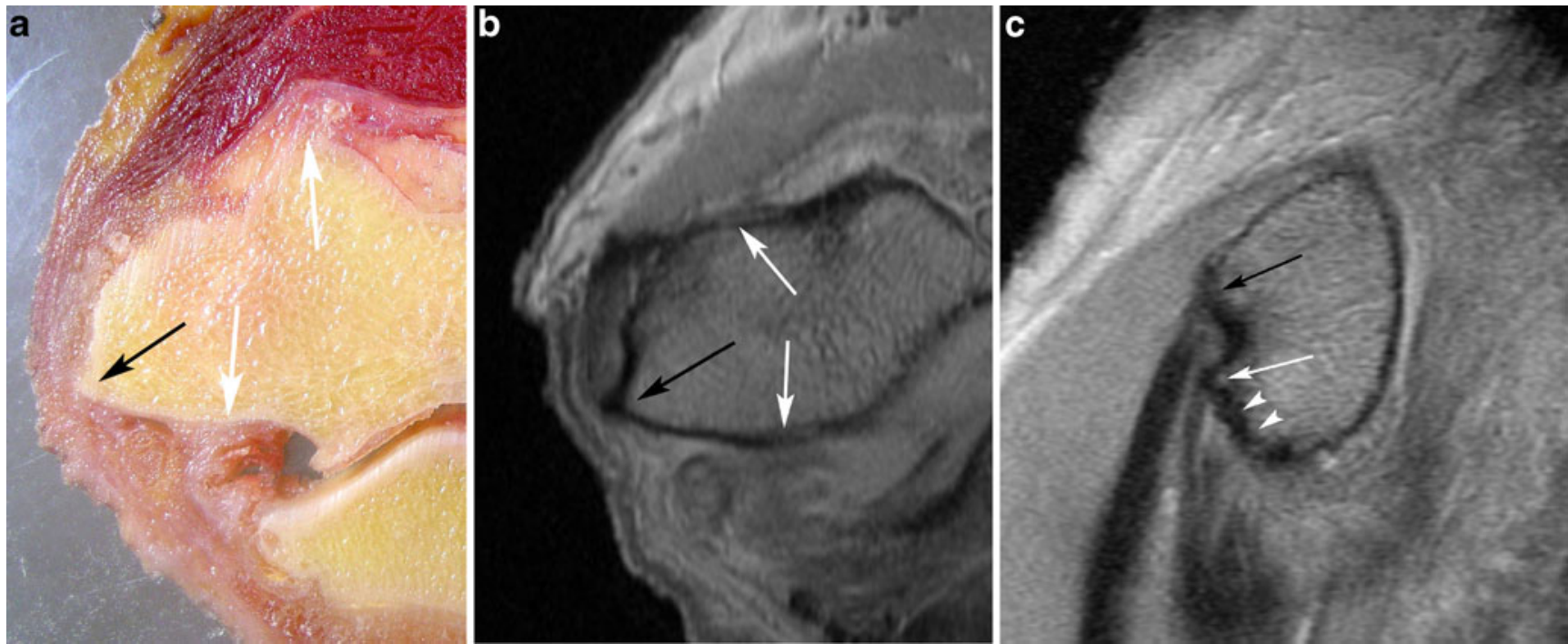

Fig. 7 a Anatomical slice in the axial plane at the level of the medial epicondyle, with a small enthesophyte (black arrow) at the epicondylar ridge. The attachment site of the joint capsule is also well seen (white arrows). b Corresponding proton density-weighted MR image. c Sagittal proton density-weighted MR Image of the same specimen with an enthesophyte at the anteroinferior tubercle (white arrow) and irregular bone surface at the inferior aspect of the epicondyle (white arrowheads). The anterosuperior tubercle is also well seen (black arrow) 
phytes found in the bone specimens and in cadaveric specimens, it appears possible that many enthesophytes are missed at the time of MR examinations.

However, since the paleopathologic specimens were very old (several hundred years), the degenerative changes could have been the result of a very different lifestyle from that of the donors of the cadaveric specimens. Thus, the prevalence of enthesophytes in paleopathologic and cadaveric specimen cannot be directly compared.

Enthesophytes may arise at the attachment site of tendons or ligaments. At the distal medial aspect of the humerus, enthesophytes at attachment sites of ligaments can arise only at the inferior epicondylar aspect because this is the only region where ligaments attach. The only exception could be the supracondylar ridge, where the ligament of Struthers, a rare accessory ligament at the medial aspect of the distal humeral shaft, attaches [20,21]. Enthesophytes at the attachment of tendons seem to be much more common at the distal medial aspect of the humerus. In our specimen we found eight-times more enthesophytes at attachment sites of tendons than at attachment sites of ligaments. In addition, all enthesophytes seen on the MR images were at attachment sites of tendons. Osteophytes were found in only the semilunar area. There were no enthesophytes that could have been mistaken for osteophytes, due to their location.

Our results were certainly limited by the fact that the identification of the attachments in the cadaveric specimens was a very difficult task. Additionally, in most specimens, the different constituents of the common flexor tendon were not identifiable. Furthermore, even though we did not find anatomical variations in our specimens, such variations would make differentiation of tendinous and ligamentous attachments even more difficult.

In conclusion, we have determined different osseous landmarks in the distal medial aspect of the humerus that can be used to differentiate between the attachments of tendons and those of ligaments. Furthermore, we have described their prevalence, identifiability, and best imaging planes. Knowledge of these landmarks at the time of interpretation of MR images of the elbow in clinical cases should aid in the assignment of a visualized abnormality to a specific tendon or ligament.

Acknowledgment This investigation was supported by the Swiss National Science Foundation and the Swiss Radiological Society.

Open Access This article is distributed under the terms of the Creative Commons Attribution Noncommercial License which permits any noncommercial use, distribution, and reproduction in any medium, provided the original author(s) and source are credited.

\section{References}

1. Morrey BF, An KN. Functional anatomy of the ligaments of the elbow. Clin Orthop Relat Res. 1985;201:84-90.

2. Fuss FK. The ulnar collateral ligament of the human elbow joint. Anatomy, function and biomechanics. J Anat. 1991;175:203-12.

3. Timmerman LA, Andrews JR. Histology and arthroscopic anatomy of the ulnar collateral ligament of the elbow. Am J Sports Med. 1994;22:667-73.

4. Beckett KS, McConnell P, Lagopoulos M, Newman RJ. Variations in the normal anatomy of the collateral ligaments of the human elbow joint. J Anat. 2000;197:507-11.

5. Morrey BF. The elbow and its disorders. 3rd ed. Philadelphia: Saunders; 2000.

6. Milz S, Tischer T, Buettner A, Schieker M, Maier M, Redman S, et al. Molecular composition and pathology of entheses on the medial and lateral epicondyles of the humerus: a structural basis for epicondylitis. Ann Rheum Dis. 2004;63:1015-21.

7. Safran MR, Baillargeon D. Soft-tissue stabilizers of the elbow. J Shoulder Elbow Surg. 2005;14(1 Suppl S):179S-85S.

8. Benjamin M, Toumi H, Ralphs JR, Bydder G, Best TM, Milz S. Where tendons and ligaments meet bone: attachment sites ('entheses') in relation to exercise and/or mechanical load. J Anat. 2006;208:471-90.

9. Resnick D, Kang HS, Pretterklieber ML. Internal derangements of joints. 2nd ed. Philadelphia: Saunders; 2006.

10. Pfirmann CWA, Zanetti M, Hodler J. Joint magnetic resonance imaging: normal variants and pitfalls related to sports injury. Radiol Clin North Am. 2002;40:167-80.

11. Mirowitz SA, London SL. Ulnar collateral ligament injury in baseball pitchers: MR imaging evaluation. Radiology. 1992;185:573-6.

12. Sugimoto H, Ohsawa T. Ulnar collateral ligament in the growing elbow: MR imaging of normal development and throwing injuries. Radiology. 1994;192:417-22.

13. Nakanishi K, Masatomi T, Ochi T, Ishida T, Hori S, Ikezoe J, et al. MR arthrography of elbow: evaluation of the ulnar collateral ligament of elbow. Skeletal Radiol. 1996;25:629-34.

14. Cotten A, Jacobson J, Brossmann J, Pedowitz R, Haghighi P, Trudell D, et al. Collateral ligaments of the elbow: conventional MR imaging and MR arthrography with coronal oblique plane and elbow flexion. Radiology. 1997;204:806-12.

15. Martin CE, Schweitzer ME. MR imaging of epicondylitis. Skeletal Radiol. 1998;27:133-8.

16. Mulligan SA, Schwartz ML, Broussard MF, Andrews JR. Heterotopic calcification and tears of the ulnar collateral ligament: radiographic and MR imaging findings. AJR Am J Roentgenol. 2001;175:1099-102.

17. Ly JQ, Sanders TG, Beall DP. MR imaging of the elbow: a spectrum of common pathologic conditions. Clin Imaging. 2005;29:278-82.

18. Jazrawi LM, Leibman M, Mechlin M, Yufit P, Ishak C, Schweitzer $\mathrm{M}$, et al. Magnetic resonance imaging evaluation of the ulnar collateral ligament in young baseball pitchers less than 18 years of age. Bull Hosp Jt Dis. 2006;63:105-7.

19. Netter FH (1989) Atlas of Human Anatomy, Ciba-Geigy Corporation, Ardsley, USA.

20. Pecina M, Boric I, Anticevic D. Intraoperatively proven anomalous Struthers' ligament diagnosed by MRI. Skeletal Radiol. 2002;31:532-5.

21. Lordan J, Rauh P, Spinner RJ. The clinical anatomy of the supracondylar spur and the ligament of Struthers. Clin Anat. 2005;18:548-51. 